\title{
Bioavailability and Bioequivalence Issues Associated With Oral Anticancer Drugs and Effect on Drug Market
}

\author{
Suryatheja Ananthula* \\ College of Pharmacy, University of Tennessee Health Sciences Center, Memphis, TN, USA
}

Since the past decade, drug shortage is becoming the major concern for pharmaceutical markets and it is even more in case of anticancer drugs $[1,2]$. Comparatively very less number of anticancer drugs is available in the current markets and various factors affecting shortage can be attributed to every stage such as drug discovery, development, conducting clinical trials and design of generic drugs. Professional health care organizations such as ASCO, ASHP and federal agencies such as FDA also stated that drug shortage will have strong negative impact on oncology $[1,2]$.These limitations are found at higher levels especially in oral anticancer drugs because of their bioavailability issues.

Oral anticancer drugs have advantage over traditional parenterally administered drugs because of their high patient acceptance rate, ease of administration and reduce a lot of patient maintenance cost. However, oral anticancer drugs should be equally effective as parental anticancer drugs and achieving this has significant limitations. Many of the anticancer drugs including camptothecins, topotecan, topoisomerase II inhibitors etoposide and teniposide, doxorubicin, anthracyclins, vinca alkaloids and other anticancer drugs are facing problems with less bioavailability and interpatient variability [3]. Moreover, several anticancer drugs have low therapeutic window and the therapeutic dose is close to maximum tolerated dose. In this case, a difference in bioavailability of the drugs due to interpatient variability is critical factor. Because of inter patient variability, the amount of drug reaching blood will be different in different individuals and it may reach maximum levels in certain patients resulting in toxic side effects $[3,4]$. Moreover, the bioavailability will also be changed in a person during the course of treatment based upon the stage of cancer or other pathological conditions. Considering the effects of inters and intra-patient variability on treatment outcome, factors such as age, sex, severity of disease condition and genotyping should be considered during studies. In addition, gastrointestinal or liver pathological conditions are not similar in between different cancer patients. These differences will alter drug absorption or metabolism in patients [3,5]. Hence, achieving desired plasma drug concentration and improving bioavailability of the drugs is challenging in different clinical conditions.

Majority of the drugs are absorbed in the intestine because of its large surface area and retention time of drug in this region. Despite their cytostatic and cytotoxic effects on the cancer cells, several anticancer drugs will also show significant cytotoxicity on healthy cells of the body depending upon the concentration in the region and exposure time. If the residence time for anticancer drugs in gastrointestinal tract is increased in a patient due to presence of food or any pathological condition associated with cancer, they may show significant adverse or toxic effects in the tract [3]. Enough care should be taken to increase absorption of these drugs from gastrointestinal tract to overcome this unwanted effects. Drug absorption is influenced by the $\mathrm{pH}$ conditions and chemical nature of the fluids at the site of absorption. Most of the developing anticancer drugs are lipophilic and they advantage of interacting well with membrane receptors of the target and pass through lipidic cell membranes [6,7]. Whereas, these cannot be solubilized in high amounts in the aqueous gastrointestinal fluids and further have low bioavailability limiting their desired anticancer effect at intended target. Even though lipophilic drugs can be transported through lymphatic system to enter into blood stream, the drug concentration in blood may not reach to therapeutic levels limiting its capacity to reach intended target [3,5].

Further, design of preclinical studies or selecting a more specific rodent cancer model is also a critical factor in developing anticancer drugs. This is due to the differences in expression of metabolizing enzymes and drug transporters between rodents and humans. Hence, assessment of bioavailability or pharmacokinetic profile of an anticancer drug in a rodent cancer model and further projecting the data from preclinical to clinical conditions is not very accurate [8]. For example, a large portion of anticancer drugs are metabolized by CYP3A group of enzymes prior to release of drug into systemic blood circulation. CYP3A group of enzymes are present both in intestine and liver. However, the expression profile and functionality of this enzyme is not similar in different patients based on the disease state and genotyping [8]. Moreover, drug efflux transporters such as P-gp are present in apical membrane of the epithelial layer of gut wall and limit hydrophilic and lipophilic drug absorption by expelling them back into intestine. Substrate specificity and tissue specific expression profiles are largely similar between CYP3A and P-gp so that they both act synergistically to decrease drug bioavailability $[5,8]$. These situations will negatively influence anticancer drug efficiency as achieving optimum drug concentration in blood is very crucial for their activity.

Drug shortage in the markets can also be overcome by manufacturing generic drugs. Generic drugs should have same active ingredient and at the same time, they should be in acceptable bioequivalent range $(20 \%)$ when compared to the original patented or brand name brand [9]. Design of generic drugs for anticancerdrugs is difficult because of the complexity in conducting BE studies. Generic anticancer drug manufacturers should consider many factors while conducting $\mathrm{BE}$ studies such as eligibility for Biopharmaceutical System (BCS) waiver, safety in healthy individuals at recommended dose, dosing regimen and therapeutic guidelines for intended patient population, PK parameters, cytotoxicity issues and more [9]. For establishing generic drug manufacturing firms is difficult due to various problems. For example, achieving high quality raw materials is very difficult and is costly for these industries. Manufacturers will also be not ready to spend high amount of money due to their low margin

*Corresponding author: Suryatheja Ananthula, College of Pharmacy, University of Tennessee Health Sciences Center, Memphis, TN, USA-38163, Tel: 901448 7161; E-mail: sananthu@uthsc.edu

Received October 14, 2014; Accepted October 15, 2014; Published October 31 2014

Citation: Ananthula S (2014) Bioavailability and Bioequivalence Issues Associated With Oral Anticancer Drugs and Effect on Drug Market. J Bioequiv Availab 6: e56. doi:10.4172/jbb.10000e56

Copyright: (c) 2014 Ananthula S. This is an open-access article distributed under the terms of the Creative Commons Attribution License, which permits unrestricted use, distribution, and reproduction in any medium, provided the original author and source are credited. 
Citation: Ananthula S (2014) Bioavailability and Bioequivalence Issues Associated With Oral Anticancer Drugs and Effect on Drug Market. J Bioequiv Availab 6: e56. doi:10.4172/jbb.10000e56

Page 2 of 2

in profits. Generic drugs also face problems because of lack of dealers, sub-standard safety monitoring techniques and low incentives from government [10].

In conclusion, we can say that shortage of orally administered anticancer drugs may hit to maximum levels in pharmaceutical markets and necessary steps needed to be taken to fill the gaps. Drug shortage will result in delay of cancer treatment and creates anxiety in both patients and health care provides.

\section{References}

1. http://www.medscape.com/viewarticle/749401.

2. http://www.medscape.com/viewarticle/733549.

3. Schellens $\mathrm{JH}$, Malingre MM, Kruijtzer CM, Bardelmeijer HA, Van Tellingen O et al. (2000) Modulation of oral bioavailability of anticancer drugs: from mouse to man. Eur J Pharm Sci 12: 103-110.

4. Wu L, Li X, Janagam DR, Lowe TL (2014) Overcoming the blood-brain barrier in chemotherapy treatment of pediatric brain tumors. Pharm Res 31: 531-540.
5. Stuuram FE, Nuijen B, BeijnenJh, Schellens JH (2013) Oral anticancer drugs: mechanisms of low bioavailability and strategies for improvement. Clin Pharmacokinet 52: 399-414.

6. Sylvester PW, AkI MR, Malaviya A, Parajuli P, Ananthula S et al. (2014) Potential role of tocotrienols in the treatment and prevention of breast cancer. Biofactors 40: 49-58.

7. Ananthula S, Parajuli P, Behery FA, Alayoubi AY, Alayoubi AY et al. (2014) Oxazine derivatives of $\gamma$ - and $\delta$-tocotrienol display enhanced anticancer activity in vivo. Anticancer Res 34: 2715-2726.

8. Bansal T, Akhtar N, Jaggi M, Khar RK, Talegaonkar S (2009) Novel formulation approaches for optimising delivery of anticancer drugs based on P-glycoprotein modulation. Drug Discov Today 14: 1067-74.

9. Kaur P, Chaurasia CS, Davit BM, Conner DP (2013) Bioequivalence study designs for generic solid oral anticancer drug products: scientific and regulatory considerations. J ClinPharmacol 53: 1252-1260.

10. Renner L, Nkansah FA, Dodoo AN (2013) The role of generic medicines and biosimilars in oncology in low-income countries. Ann OncolSuppl 5: 29-32. 